\title{
Contribution to the knowledge of seed-beetles (Coleoptera, Chrysomelidae, Bruchinae) in Xinjiang, China
}

\author{
You Li ${ }^{1,2}$, Zhiliang Wang', Jianjun Guo ${ }^{3}$, Jesús Romero Nápoles ${ }^{4}$, \\ Yingchao Ji', ${ }^{1,5}$ Chunyan Jiang', Runzhi Zhang'
}

I Key Laboratory of Zoological Systematics and Evolution, Institute of Zoology, Chinese Academy of Sciences, Beijing 100101, China 2 School of Forest Resources and Conservation, University of Florida, Gainesville, FL 32611, USA 3 Institute of Entomology, Guizhou University, The Provincial Key Laboratory for Agricultural Pest Management of Mountainous Region, Guiyang 550025, China 4 Instituto de Fitosanidad, Colegio de Postgraduados, km 36.5 carr. Fed. México-Texcoco, Montecillo, Estado de México, C.P. 56230, México 5 College of Plant Protection, Shandong Agricultural University, Tai'an 271018, China

Corresponding author: Runzhi Zhang (zhangrz@ioz.ac.cn)

Academic editor:A. Konstantinov| Received 18February2014|Accepted3 December 2014| Published 18December 2014

http://zoobank.org/3B768ACB-2384-43FA-BD2F-B41328052FC8

Citation: Li Y, Wang Z, Guo J, Nápoles JR, Ji Y, Jiang C, Zhang R (2014) Contribution to the knowledge of seed-beetles (Coleoptera, Chrysomelidae, Bruchinae) in Xinjiang, China. ZooKeys 466: 13-28. doi: 10.3897/zookeys.466.7283

\begin{abstract}
Nineteen species of seed-beetles belonging to the subfamily Bruchinae (Coleoptera, Chrysomelidae) were collected in Xinjiang, China. Of these, the following four were new records for China: Bruchus affinis Frolich, 1799, B. atomarius L., 1761, B. loti Paykull, 1800 and Kytorhinus kergoati Delobel \& Legalov, 2009. We provide an annotated checklist, illustrations and a key to the 19 species.
\end{abstract}

\section{Keywords}

New record, checklist, taxonomy, Palaearctic region

\section{Introduction}

Xinjiang Uygur Autonomous Region (hereafter referred to as Xinjiang, also known as Sinkiang) is a provincial region in the northwest of the People's Republic of China. It is the largest Chinese administrative division and it spans over 1.6 million $\mathrm{km}^{2}$. The

Copyright You Li et al. This is an open access article distributed under the terms of the Creative Commons Attribution License (CC BY 4.0), which permits unrestricted use, distribution, and reproduction in any medium, provided the original author and source are credited. 
region is bordered by eight countries: Russia, Mongolia, Kazakhstan, Kyrgyzstan, Tajikistan, Afghanistan, Pakistan and India.

Four tribes of the Chrysomelidae subfamily Bruchinae have been recorded in Xinjiang: Amblycerini, Bruchini, Kytorhinini and Rhaebini (Anton 2010, Tan and Yu 1980) and six tribes according to Bouchard et al. (2011): Amblycerini, Bruchini, Eubaptini, Kytorhinini, Pachymerini and Rhaebini. Kytorhinini and Rhaebini are monotypic and restricted to Central Asia and the temperate Holarctic region, respectively (Borowiec 1987, Delobel and Legalov 2009). The majority of Bruchinae species, commonly called bean weevils or seed-beetles, feed on grain legumes and seeds of leguminous trees and shrubs. Many species have a significant economic impact because they can consume valuable protein-rich crops that would otherwise be eaten by humans (Southgate 1979). Despite this, little is currently known about the seed-beetles of Xinjiang. Fifteen species have been documented (Hoffmann 1965, Tan and Yu 1980, Zhang et al. 1987, Xu 1991, Anton 2010, Sui et al. 2011), but most of the records lack detailed information about their distribution.

\section{Methods}

We checked all seed-beetles specimens from Xinjiang (most collected from 1956 to 1978) in National Zoological Museum of China (NZMC), Institute of Zoology, Chinese Academy of Science (IZCAS), in Beijing, China. In order to increase the material currently available in the NZMC collection, we collected twice in Xinjiang in July 2009 and August 2013. Seed-beetles were obtained in the field by sweeping with a sweep net and by collecting seeds of host plants in day time. We also tried to collect at night, but no seed-beetles were found. The identification of some of the specimens were confirmed by Chinese Chrysomelidae specialist Tan Juanjie and Yu Peiyu of IZCAS twenty years ago. All the specimens were identified by comparing the external morphological features and the male genitalia morphological characters with some published articles again (Lukjanovitsch and Ter-Minassian 1957, Tan and Yu 1980, Borowiec 1987, 1991, Kingsolver 2004, Delobel and Legalov 2009).

Photographs of all the seed-beetles were taken with a Cannon 5D digital camera and images were processed in Adobe Photoshop CS5. Drawings were created using Adobe Illustrator CS4. All specimens were deposited in the NZMC, where most of them were assigned unique numbers corresponding to the Institute of Zoology collection code entry $\mathrm{IOZ}(\mathrm{E})$.

\section{Results}

In this study, 19 species of Bruchinae beetles were collected in Xinjiang. They were annotated with updated detailed distribution in the following checklist. The following key is illustrated with photographs of morphological characters used in it. 


\section{Checklist of Bruchinae from Xinjiang, China}

Tribe Amblycerini Bridwell, 1932

Subtribe Spermophagina Borowiec, 1987

Genus Spermophagus Schoenherr, 1833

Spermophagus sericeus (Geoffroy, 1785)

Figs 1-2

Material. 20, Akqi, Kizilsu, Xinjiang, $40.98^{\circ} \mathrm{N}, 78.70^{\circ} \mathrm{E}$, alt. ca $1970 \mathrm{~m}, 2005 . V I .14$, H.Y. Hu leg.; $1960^{\lambda}$, Aksu, Xinjiang, $40.94^{\circ} \mathrm{N}, 80.11^{\circ} \mathrm{E}$, alt. ca $1180 \mathrm{~m}, 1978 . V I .19$, Y.H. Han leg.; IOZ(E)1016347-1016583; 19, Qinggil, Altay, Xinjiang, $46.69^{\circ} \mathrm{N}$,

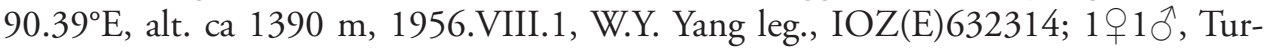
pan, Xinjiang, $42.93^{\circ} \mathrm{N}, 89.27^{\circ} \mathrm{E}$, alt. ca $140 \mathrm{~m}, 1958 . V .20$, C.Q. Li and G. Wang leg., IOZ(E)632431, IOZ(E)632433; 3 우을, Baicheng, Aksu, $41.78^{\circ} \mathrm{N}, 81.92^{\circ} \mathrm{E}$, alt. ca 1310 m, 1959.VII.22, A.F. Tian leg., IOZ(E)115170-115176; 1우스, Yuli, Mila, Bayingol, Xinjiang, $41.77^{\circ} \mathrm{N}, 84.24^{\circ} \mathrm{E}$, alt. ca $1000 \mathrm{~m}, 1958$.VII.13, C.Q. Li

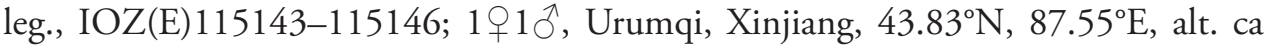
820 m, 1955.VII.25, S.J. Ma, K.L. Xia and Y.L.Chen leg., IOZ(E)115133-115134;

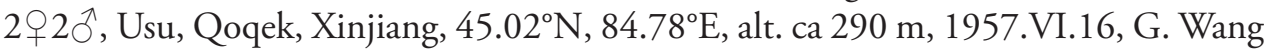
leg., IOZ(E)115106-115109; $10+16{ }^{\lambda}$, Shihezi, Xinjiang, $44.28^{\circ} \mathrm{N}, 86.27^{\circ} \mathrm{E}$, alt. ca 500 m, 1957.VI.7, G. Wang and C.P. Hong leg., IOZ(E)115080-115106; 1q6ð, Shawan, Qoqek, Xinjiang, $44.46^{\circ} \mathrm{N}, 85.66^{\circ} \mathrm{E}$, alt. ca $420 \mathrm{~m}, 1957 . V I .11, \mathrm{G}$. Wang and C.P. Hong leg., IOZ(E)115063-115169; 1 \% , Jeminay, Altay, Xinjiang, $47.43^{\circ} \mathrm{N}$, $85.87^{\circ} \mathrm{E}$, alt. ca $970 \mathrm{~m}, 1956 . \mathrm{IX} .17$, W.Y. Yang leg., IOZ(E) 115054.

Distribution. Widely distributed around the Palaearctic region.

Tribe Bruchini Latreille, 1802

Subtribe Acanthoscelidina Bridwell, 1946

Genus Acanthoscelides Schilsky, 1905

Acanthoscelides pallidipennis (Motschulsky, 1874)

Figs 3-4, 49

Distribution. Armenia, Austria, Azerbaijan, Bulgaria, China, Croatia, Czech Republic, France, Germany, Greece, Hungary, Italy, Japan, Macedonia, North America, North Korea, Russia, Slovakia, Switzerland, Serbia and Montenegro, Tajikistan.

Remarks. We did not find any specimens of A. pallidipennis in Xinjiang in our study, however Tan and $\mathrm{Yu}(1980)$ recorded it in Xinjiang. According to Tan and $\mathrm{Yu}$ (1980), the North American bruchid A. pallidipennis was introduced to China with its natural host Amorpha fruticosa L. a number of years ago. It has been a major pest of $A$. fruticosa seeds in China. 


\section{Genus Bruchidius Schilsky, 1905}

\section{Bruchidius apicipennis Heyden, 1892}

Figs 5-6

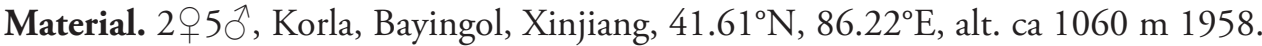
VIII.11-17, C.Q. Li, IOZ(E)109480-109482, 109484, 109486, 109488-109489; 10, Aksu, Xinjiang, $41.18^{\circ} \mathrm{N}, 80.19^{\circ} \mathrm{E}$, alt. ca $1210 \mathrm{~m}, 1958 . \mathrm{IX} .9$, C.Q. Li, IOZ(E)109517;

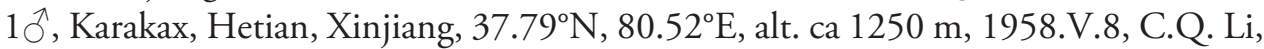
$\operatorname{IOZ}(\mathrm{E}) 109516 ; 12{ }^{\wedge} 13$ 으, Halajunxiang, Artux, Kizilsu, Xinjiang, $40.02^{\circ} \mathrm{N}, 76.81^{\circ} \mathrm{E}$, alt. ca 1610 m, 1959.VI.22, S.Y. Wang leg., IOZ(E)109490-109514; 10, Yanqi, Bayingol, $41.80^{\circ} \mathrm{N}, 85.82^{\circ} \mathrm{E}$, alt. ca $950 \mathrm{~m}, 1958$.VIII.26, C.Q. Li, IOZ(E)109515; 19 , Hetian, Xinjiang, $37.02^{\circ} \mathrm{N}, 79.98^{\circ} \mathrm{E}, 1955$. V.20, S.J. Ma, K.L. Xia and Y.L. Chen leg., IOZ(E) $109657 ; 2$, Jinghe, Bortala, Xinjiang, $44.36^{\circ} \mathrm{N}, 83.15^{\circ} \mathrm{E}$, alt. ca 1730 m, 1955.VIII.24, S.J. Ma, K.L. Xia and Y.L. Chen leg., IOZ(E)109518-109519; 1 \%, Manas, Changji, Xinjiang, $44.54^{\circ} \mathrm{N}, 86.22^{\circ} \mathrm{E}$, alt. ca $400 \mathrm{~m}, 1957 . V I .9$, G. Wang, IOZ(E)109521; $4{ }^{\lambda} 3$ ㅇ, Milan, Ruoqiang, Bayingol, Xinjiang, $39.27^{\circ} \mathrm{N}, 89.10^{\circ} \mathrm{E}$, alt. ca 900 m 1960.IV.30, S.Y. Wang leg., IOZ(E)109649-109655; 2ㅇ, Xiao Artux, Artux, Kizilsu, Xinjiang, $39.68^{\circ} \mathrm{N}, 75.67^{\circ} \mathrm{E}$, alt. ca $2100 \mathrm{~m}, 1959 . V I .17$, S.Y. Wang leg., IOZ(E)109658-109659; 1우, Shihutang, Manas, Changji, Xinjiang, $44.60^{\circ} \mathrm{N}$, $86.09^{\circ} \mathrm{E}$, alt. ca $370,1957 . V I I .4$, C.P. Hong leg., IOZ(E)109660; $1 \overbrace{}^{\wedge} 2$ 어, Wensu, Aksu, Xinjiang, $41.29^{\circ} \mathrm{N}, 80.21^{\circ} \mathrm{E}$, alt. ca $1190,1955 . V I .9$, S.J. Ma, K.L. Xia and Y.L. Chen leg., IOZ(E)109841-109843.

Distribution. China, Iran, Kazakhstan, Mongolia, Russia, South Africa, Turkey, Turkmenistan.

\section{Bruchidius tuberculicauda Lukjanovitsch \& Ter-Minassian, 1954}

Figs 7-8, 64-65

Material. 1 우 $10^{\Uparrow}$, Nilka, Ila, Xinjiang, $43.79^{\circ} \mathrm{N}, 82.50^{\circ} \mathrm{E}, 1124 \mathrm{~m}, 1994 . V I .20$, X.F. Huang leg.; 2 ㅇ $1 \hat{O}^{\hat{\prime}}$, Takeshikenzhen, Qinggil, Altay, Xinjiang, $46.18^{\circ} \mathrm{N}, 90.81^{\circ} \mathrm{E}$, alt. ca 1110 m, 2013.VII.28, Y. Li leg..

Distribution. China, Kyrgyzstan, Kazakhstan, Mongolia, Russia.

\section{Genus Callosobruchus Pic, 1902}

\section{Callosobruchus chinensis (L., 1975)}

Figs 9-10

Material. 1 , Xinhe, Xinjiang, $41.51^{\circ} \mathrm{N}, 82.50^{\circ} \mathrm{E}$, alt. ca $980 \mathrm{~m}, 2000 . \mathrm{VI} .30$, R.H. Lin leg. 


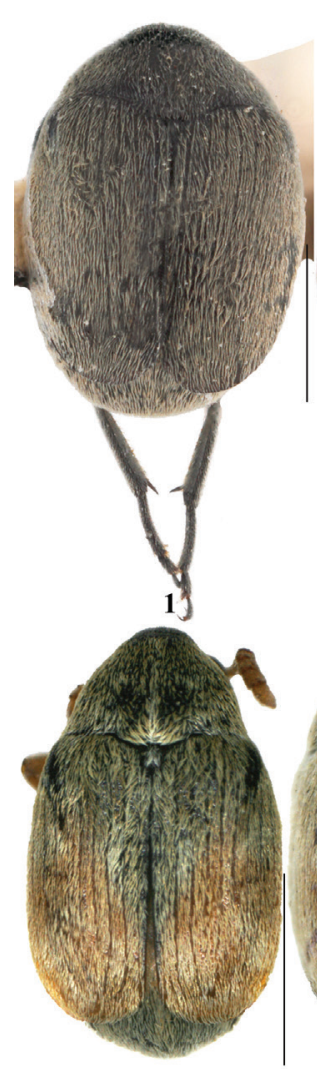

5

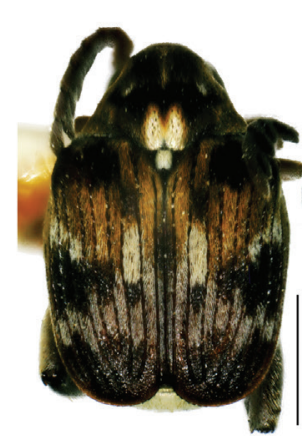

9
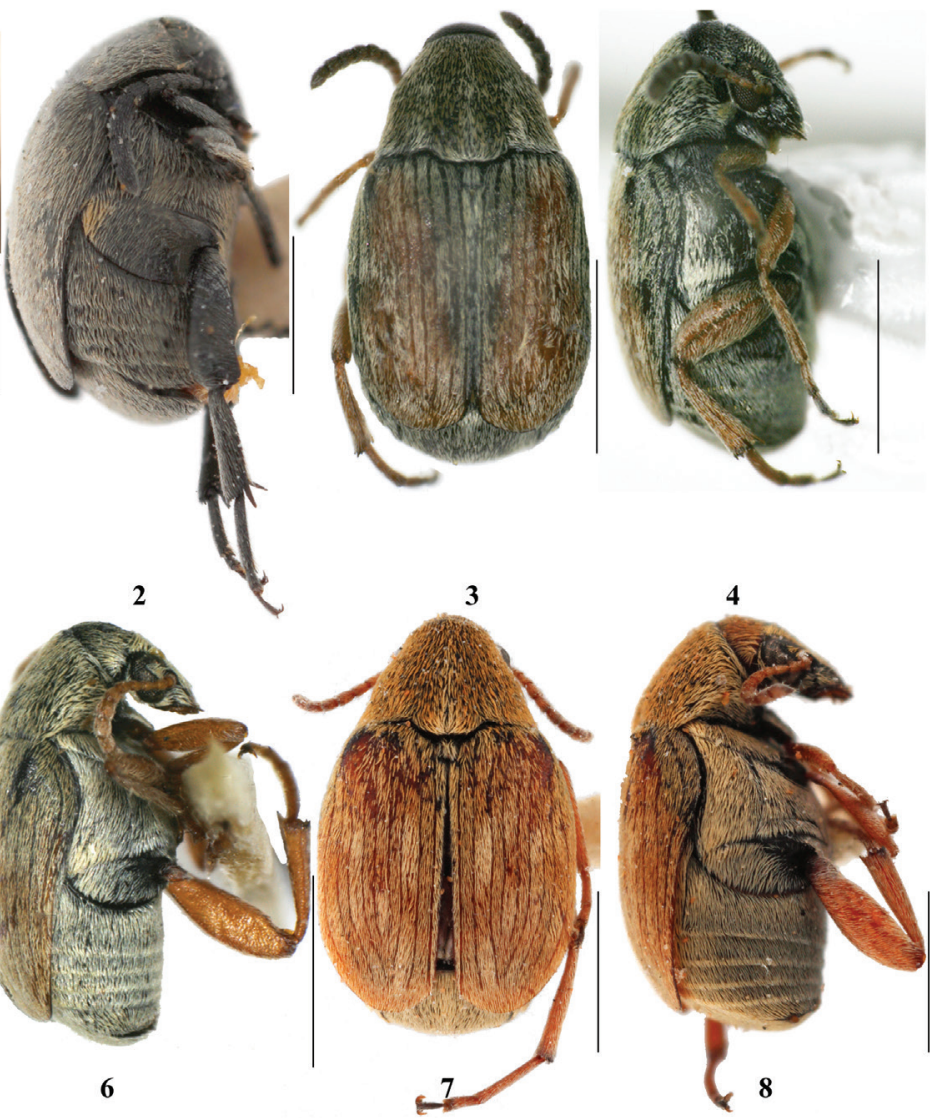

6

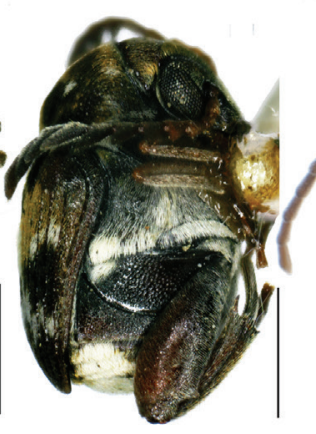

10

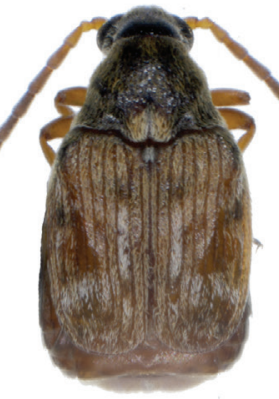

11

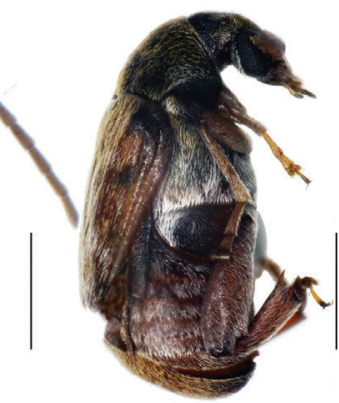

12

Figures I-12. Bruchinae in Xinjiang, dorsal and lateral view. I-2 Spermophagus sericeus 3-4 Acanthoscelides pallidipennis 5-6 Bruchidius apicipennis 7-8 B. tuberculicauda 9-10 Callosobruchus chinensis I I-I 2 C. maculatus; black bar $=1 \mathrm{~mm}$.

Distribution. Almost worldwide.

Remarks. In this study, we found only one specimen of $C$. chinensis in Xinjiang. Zhang et al. (1987) and Xu (1991), however, recorded Vigna radiata and V. angularis extensively infested by C. chinensis in Kumul and Shihezi, Xinjiang. 


\section{Callosobruchus maculatus (Fabricius, 1975)}

Figs 11-12, 50-51

Distribution. Almost worldwide.

Remarks. Although we did not collect any C. maculatus specimens from Xinjiang in this study, Sui et al. (2001) previously recorded Cicer arietinum infested by C. maculatus in Kashgar City, Xinjiang.

\section{Genus Megabruchidius Borowiec, 1984}

\section{Megabruchidius dorsalis (Fabraeus, 1839)}

Figs 13-14, 66-67

Material. $1095 \hat{\partial}^{\lambda}$, Ili Forestry Science Research Institute, Gulja, Ili, Xinjiang, $43.94^{\circ} \mathrm{N}$, $81.33^{\circ} \mathrm{E}$, alt. ca $660 \mathrm{~m}, 1973$.VII.5, IOZ(E)109814-109818, 632556-632565.

Distribution. Bulgaria, China, France, Greece, Hong Kong, Hungary, India, Italy, Japan, Mongolia, Papua New Guinea, Switzerland, Turkmenistan.

Subtribe Bruchina Latreille, 1802

Genus Bruchus L., 1767

Bruchus affinis Frolich, 1799

Figs 15-16, 52, 59, 68

Material. $793{ }^{\top}$, Xinyuan, Ili, Xinjiang, $43.42^{\circ} \mathrm{N}, 82.26^{\circ} \mathrm{E}$, alt. ca $1200 \mathrm{~m}, 1972 . \mathrm{VII}$, IOZ(E)1016073-1016075, 108162-108163, 108157-108160, 108155.

Distribution. China, Afghanistan, Kyrgyzstan, Kazakhstan, Lebanon, Mongolia, North Korea, Russia, Syria, Tajikistan, Europe.

Bruchus atomarius (L., 1761)

Figs $17-18,53,63,68$

Material. $5920^{\lambda}$, Xinyuan, Ili, Xinjiang, $43.42^{\circ} \mathrm{N}, 82.26^{\circ} \mathrm{E}$, alt. ca $1200 \mathrm{~m}, 1972 . \mathrm{VII}$, IOZ(E)1016068-1016072, 108161, 108156; 1운 Kanasi, Buerjin County, Altay, Xinjiang, $49.01^{\circ} \mathrm{N}, 87.35^{\circ} \mathrm{E}$, alt. ca $1550 \mathrm{~m}, 2009$.VII.25, Z.L. Wang leg.

Distribution. New record for China, Europe, Iran, Kyrgyzstan, Kazakhstan, Lebanon, Mongolia, North Korea, Russia, Syria. 

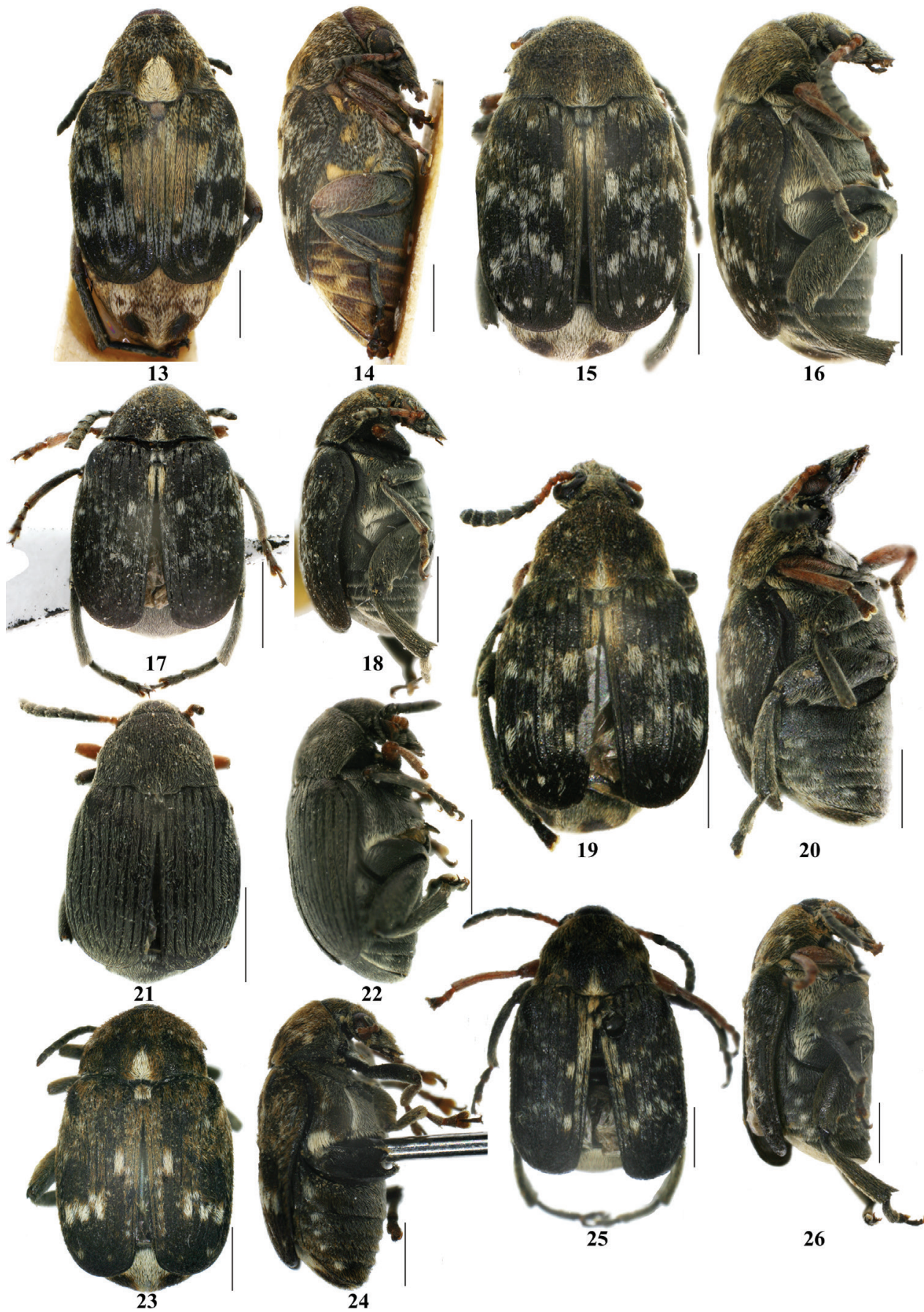

Figures 13-26. Bruchinae in Xinjiang, dorsal and lateral view. I 3-I 4 Megabruchidius dorsalis I 5- 16 Bruchus affinis 17-1 8 Bruchus atomarius 19-20 Bruchus dentipes 21-22 Bruchus loti 23-24 Bruchus pisorum 25-26 Bruchus rufimanus; black bar $=1 \mathrm{~mm}$. 


\section{Bruchus dentipes Baudi, 1886}

Figs 19-20, 54

Distribution. Afghanistan, Algeria, Azerbaijan, Armenia, Belgium, China, Croatia, Cyprus, Egypt, England, France, Greece, Italy, Iran, Iraq, Israel, Jordan, Kazakhstan, Lebanon, Russia, Spain, Switzerland, Syria, Tajikistan, Turkmenistan, Turkey, Uzbekistan.

Remarks. We did not collect any $B$. dentipes specimens in Xinjiang in this study, but Tan and Yu (1980) previously recorded $B$. dentipes as occurring in Xinjiang.

\section{Bruchus loti Paykull, 1800}

Figs 21-22, 55, 68

Material. 3 , , Xinyuan, Ili, Xinjiang, $43.42^{\circ} \mathrm{N}, 82.26^{\circ} \mathrm{E}$, alt. ca $1200 \mathrm{~m}, 1972 . \mathrm{VII}$, IOZ(E) 1016065-1016067.

Distribution. Algeria, New record for China, Eurasia, Japan, Morocco, Russia, Turkey, Ukraine.

Bruchus pisorum (L., 1758)

Figs 23-24, 56, 60

Distribution. Worldwide.

Remarks. In this study, we did not find any B. pisorum specimens in Xinjiang, but Yixin (1991) previously recorded B. pisorum as occurring in Xinjiang.

\section{Bruchus rufimanus Boheman, 1833}

Figs 25-26, 46, 57, 62

Distribution. Worldwide except Australia.

Remarks. We did not find any B. rufimanus specimens in Xinjiang; however it was previously recorded as occurring there (Tan and Yu 1980).

\section{Bruchus sibiricus Germar, 1824}

Figs 27-28, 58, 61

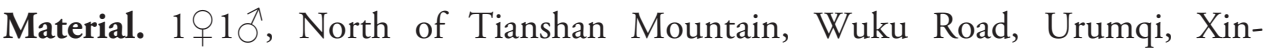
jiang, $43.56^{\circ} \mathrm{N}, 87.19^{\circ} \mathrm{E}$, alt. ca $1600 \mathrm{~m}, 1960$. VI.11, S.Y. Wang leg., IOZ(E) $1045200-1045201$.

Distribution. Azerbaijan, Armenia, China, Kyrgyzstan, Kazakhstan, Mongolia, Russia, Tajikistan, Turkey, Uzbekistan. 


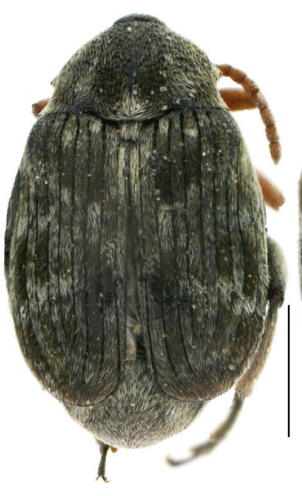

27

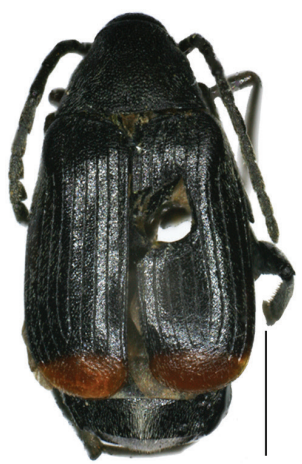

31

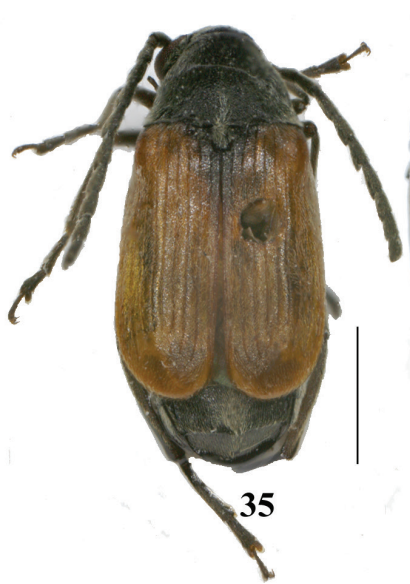

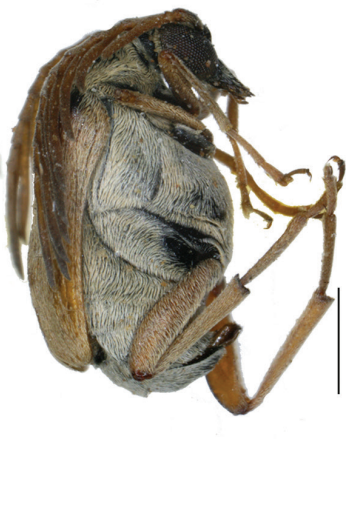

30

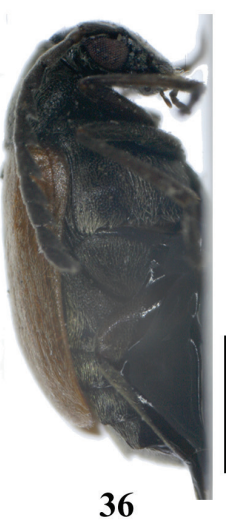

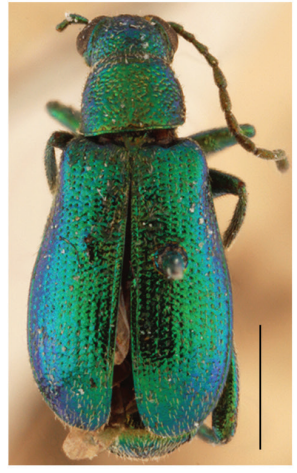

37

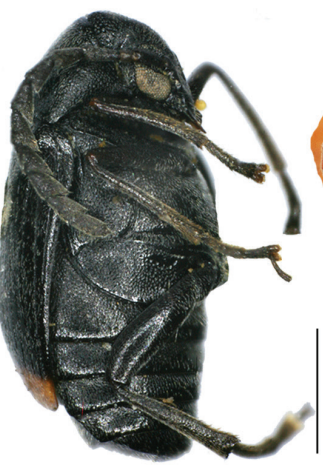

32

33

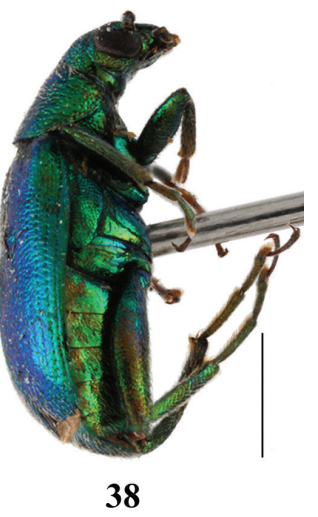

Figures 27-38. Bruchinae in Xinjiang, dorsal and lateral view. 27-28 Bruchus sibiricus 29-30 Kytorhinus immixtus 3 I-32 Kytorhinus karasini 33-34 Kytorhinus thermopsis 35-36 Kytorhinus kergoati 37-38 Rhaebus solskyi; black bar $=1 \mathrm{~mm}$. 
Tribe Kytorhinini Bridwell, 1932

Genus Kytorhinus Fischer von Waldheim, 1809

\section{Kytorbinus immixtus Motschulsky, 1874}

Figs 29-30, 43

Material. $1 \delta^{\lambda}$, Pochengzi, Wensu, Aksu, Xinjiang, $41.77^{\circ} \mathrm{N}, 80.99^{\circ} \mathrm{E}$, alt. ca $2000 \mathrm{~m}$, 1978.VI.15.

Distribution. China, Kyrgyzstan, Russia.

\section{Kytorbinus karasini Fischer, 1809}

Figs 31-32

Material. 1엉 Tianshan Mountain, Fukang, Changji, Xinjiang, $43.95^{\circ} \mathrm{N}, 88.15^{\circ} \mathrm{E}$, alt. ca 2150 m, 1957.VII.14, G. Wang leg., IOZ(E)115178; 1 9 , Tianshan Mountain, Fukang, Changji, Xinjiang, $43.95^{\circ} \mathrm{N}, 88.40^{\circ} \mathrm{E}$, alt. ca $2250 \mathrm{~m}$, 1957.VII.14, G. Wang leg., IOZ(E) 107522 ; 1 , Z Zhao Su Xian, Yili, Xinjiang, $42.66^{\circ} \mathrm{N}, 80.18^{\circ} \mathrm{E}$, alt. ca 2200 m, 1978.VIII.7, Y.H. Han leg., IOZ(E)1045574; 1 , Takeshikenzhen, Qinggil, Altay, Xinjiang, $46.18^{\circ} \mathrm{N}, 90.81^{\circ} \mathrm{E}$, alt. ca $1110 \mathrm{~m}, 2013$.VII.28, Y. Li leg.

Distribution. China, Russia.

\section{Kytorhinus kergoati Delobel \& Legalov, 2009}

Figs 35-36, 41-42, 68

Material. 1 울 Pochengzi, Wensu, Aksu, Xinjiang, $41.77^{\circ} \mathrm{N}, 80.91^{\circ} \mathrm{E}$, alt. ca 1930 m, 1978.VI.3, X.Z. Zhang leg., IOZ(E)1045598; 1910, Tomur peak, Wensu, Aksu, Xinjiang, $41.74^{\circ} \mathrm{N}, 80.58^{\circ} \mathrm{E}$, alt. ca $2400 \mathrm{~m}, 1977 . V I .19$, C.J. Li leg., IOZ(E)632265-632266; 1 ${ }^{\lambda}$, Tomur peak, Wensu, Aksu, Xinjiang, $41.81^{\circ} \mathrm{N}$, $80.59^{\circ} \mathrm{E}$, alt. ca $3200 \mathrm{~m}$, 1977.VII.11, Y.H. Han leg., IOZ(E)632264; 10, Tomur peak, Wensu, Aksu, Xinjiang, 41.81 N, 80.59 E, alt. ca 3200 m, 1977.VII.14, C.J. Li leg., IOZ(E)632263; 10, Yangbajingzhen, Damxung, Lhasa, Tibet, China, $30.02^{\circ} \mathrm{N}$, $90.39^{\circ} \mathrm{E}$, alt. ca $4310 \mathrm{~m}, 1960$. VI.2, C.G. Wang leg., IOZ(E) 1016165.

Distribution. New record for China, Tajikistan (Gorno-Badakhshan autonomous province).

Host. Unknown.

Remarks. Delobel and Legalov (2009) described this species based on a single male. We examined a female specimen and found that its antennae are serrated and are slightly longer than half of the body length (Fig. 35, 41, 42). Additionally, the elytra of the female are almost yellow, with the exception of a black, elongate triangular area, which extends from the base to one-third of the elytral suture (Fig. 35) corresponding to main distinguishing characters of a single known male of $K$. kergoati. 

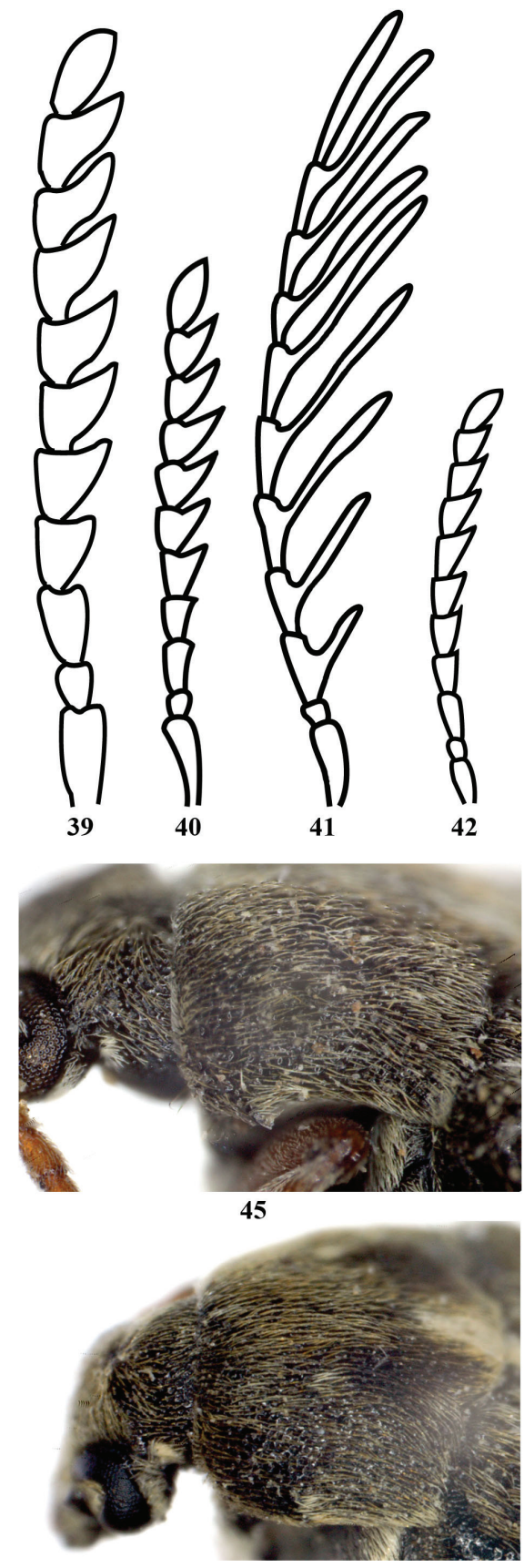

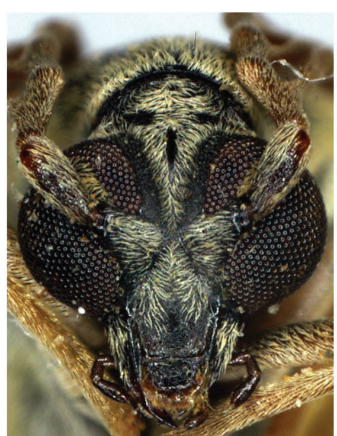

43

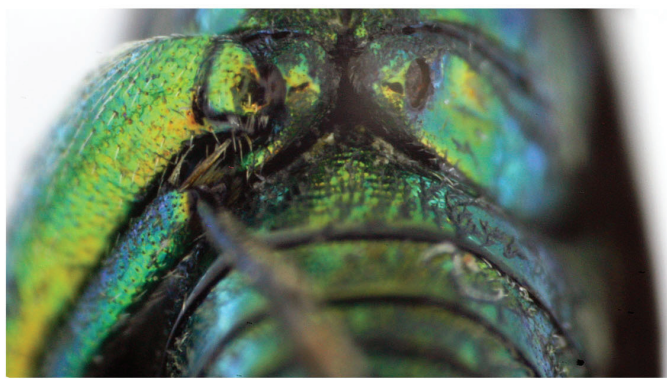

47
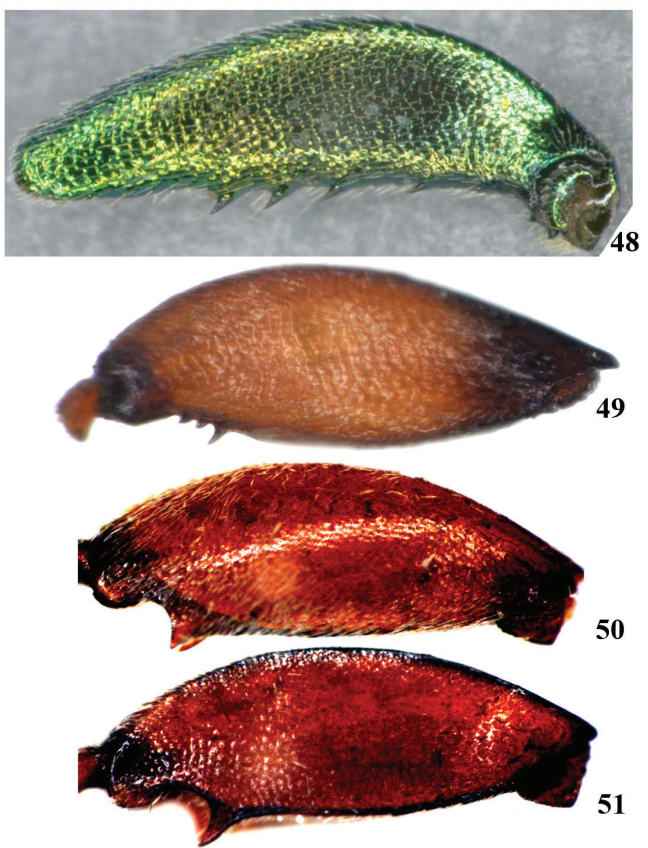

Figures 39-42. Antennae. 39 Kytorhinus thermopsis male $40 \mathrm{~K}$. thermopsis female 41 Kytorhinus kergoati male $\mathbf{4 2}$ Kytorhinus kergoati female 43-44 Head $\mathbf{4 3}$ Kytorhinus immixtus $\mathbf{4 4}$ Kytorhinus thermopsis 45-46 Lateral pronotal margin. 45 Bruchus affinis 46 Bruchus rufimanus 47 Hind trochanters 47 Rhaebus solskyi 48-5I Hind femur. 48 Rhaebus solskyi male 49 Acanthoscelides pallidipennis 50 Callosobruchus maculatus outside view $\mathbf{5}$ I Callosobruchus maculates inside view. 

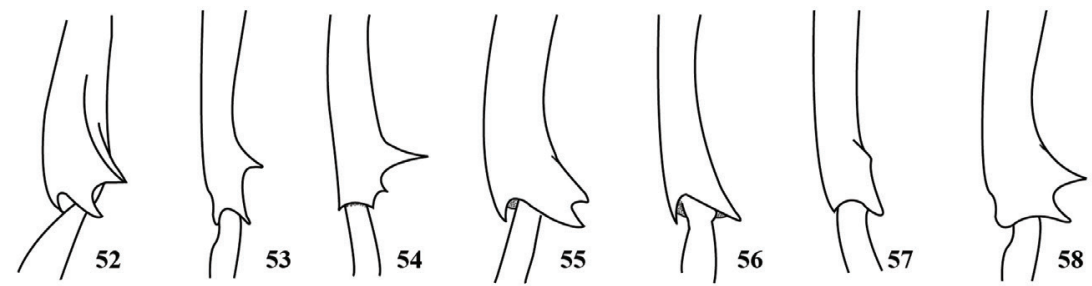

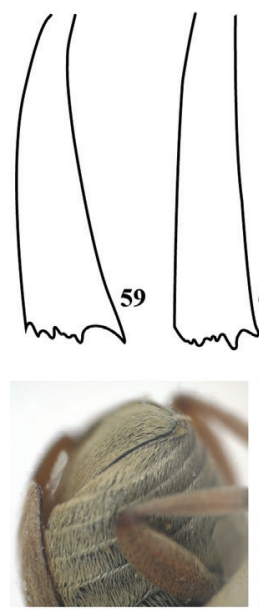

64

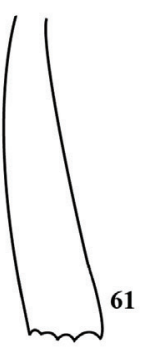

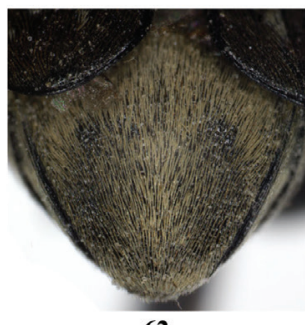

62

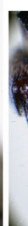

65

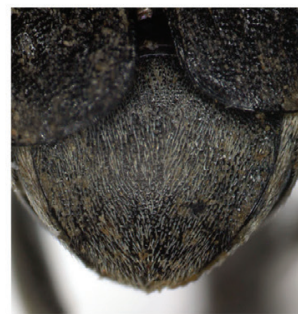

63

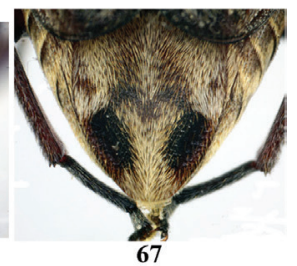

Figures 52-68. Tibia apical portion of Bruchus spp. male mid legs. 52 Bruchus affinis 53 Bruchus atomarius 54 Bruchus dentipes 55 Bruchus loti 56 Bruchus pisorum 57 Bruchus rufimanus 58 Bruchus sibiricus 59-6I Tibia apical portion of Bruchus spp. hind legs 59 Bruchus affinis 60 Bruchus pisorum 6 I Bruchus sibiricus 62-67 Pygidium. 62 Bruchus rufimanus 63 Bruchus atomarius 64 Bruchidius tuberculicauda male 65 Bruchidius tuberculicauda female 66 Megabruchidius dorsalis male 67 Megabruchidius dorsalis female.

\section{Kytorhinus thermopsis Motschulsky, 1874}

Figs 33-34, 39, 40

Material. 1 , , Xinjiang, IOZ(E) $115177 ; 19,6$ km northwest of Fuyun, Altay, Xinjiang, $47.14^{\circ} \mathrm{N}, 87.55^{\circ} \mathrm{E}$, alt. ca $650 \mathrm{~m}, 2009$.VII.13, X.L. Huang leg.

Distribution. China, Kazakhstan, Mongolia, Russia.

\section{Tribe Rhaebini Blanchard, 1845}

\section{Genus Rhaebus Fischer von Waldheim, 1824}

\section{Rhaebus solskyi Kraatz, 1879}

Figs 37-38, 47-48

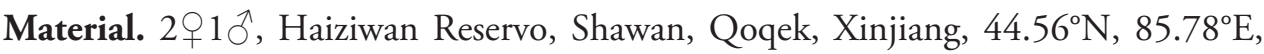
alt. ca 390 m, 1957.VI.9, C.P. Hong leg., IOZ(E)107501-107503; 1 9 , Takeshikenzhen, Qinggil, Altay, Xinjiang, $46.18^{\circ} \mathrm{N}, 90.81^{\circ} \mathrm{E}$, alt.1110 m, 2013.VII.28, Y. Li leg.

Distribution. China, Kazakhstan, Mongolia, Russia. 


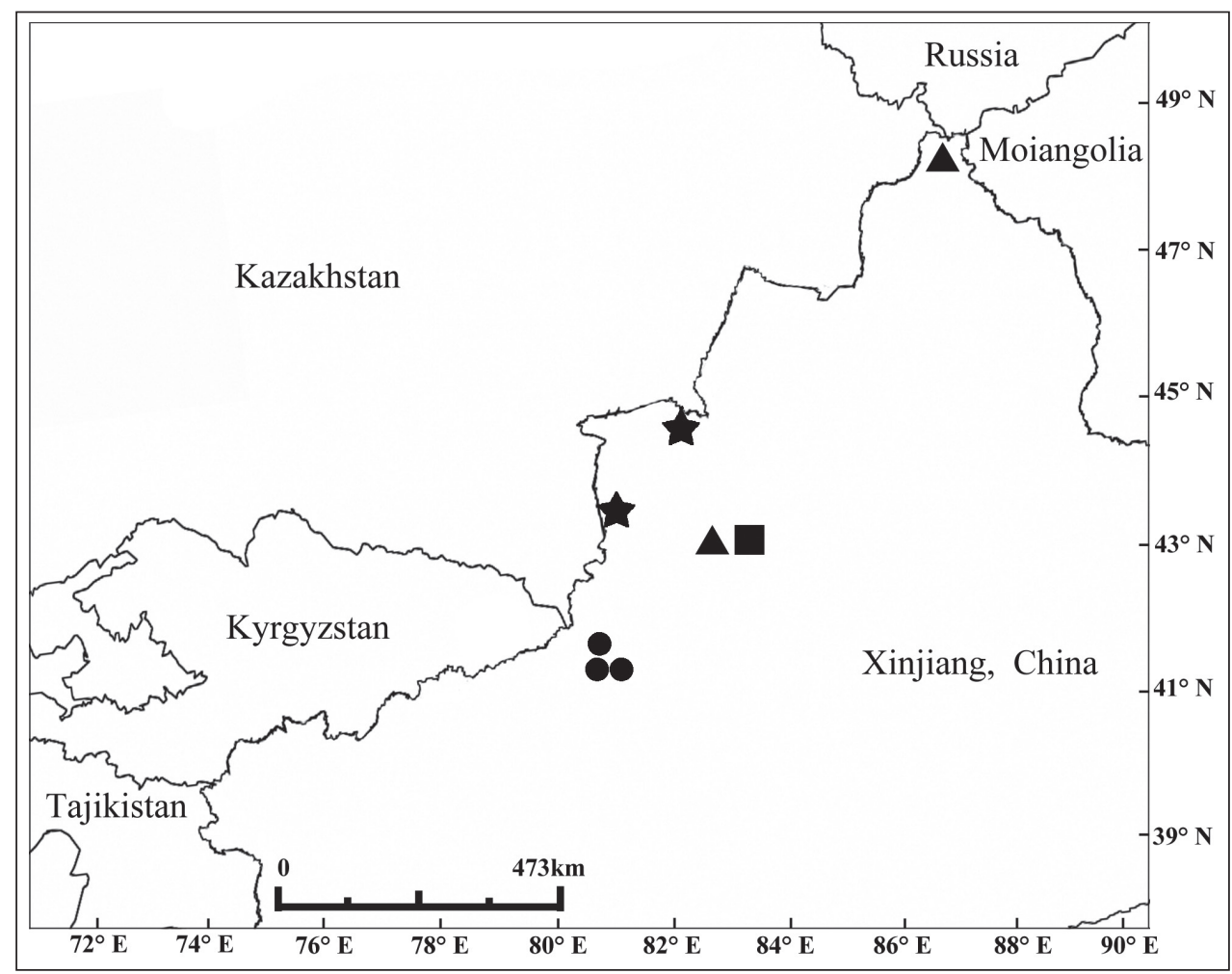

Figure 68. Map of northwestern China, illustrating localities for Bruchinae species. Bruchus affinis and Bruchus lot $i=$ squares. Bruchus atomarius $=$ triangles. Kytorhinus kergoat $i=$ dots. Only new records reported here are shown.

\section{Key to species of Bruchinae in Xinjiang}

$1 \quad$ Body completely metallic in color (Fig. 37); hind trochanters extremely enlarged (Fig. 47); hind femur with 3-8 small, evenly spaced spines on ventral side (Fig. 48).

Rhaebus solskyi

- $\quad$ Body not metallic in color; hind trochanters small; hind femur without 3-8 small, evenly spaced spines on ventral side

2 Antennae sexually dimorphic, male antennae strongly serrate (Fig. 39) or pectinate (Fig. 41), female antennae moderately serrate (Figs 40, 42); 3 last abdominal tergites (including pygidium) exposed behind the elytra (Figs 29, $31,33,35)$

- $\quad$ Antennae not sexually dimorphic, sometimes male and female antennae with different color; only pygidium exposed behind the elytra (Figs 62-67) .......6

$3 \quad$ Elytra integument with single color (Figs 29, 33) ................................ 4

- $\quad$ Elytra integument with two colors (Figs 31, 35) ....................................5

$4 \quad$ Antennae, legs and elytra integument yellow (Fig. 29); eyes large and separated by 0.2 times head width including eyes (Fig. 43) 
Antennae and legs reddish brown, elytra integument black (Fig. 33); eyes medium-sized and separated by 0.4 times head width including eyes (Fig. 44) Kytorbinus thermopsis Antennae and legs black, elytra integument almost yellow except by black basal area elongated, triangular (Fig. 35) Kytorbinus kergoati Body almost black, only apex of elytra red (Fig. 31).... Kytorhinus karasini Lateral pronotal margins with tubercle (maybe obscured by setae) (Figs 45, 46); mesotibia at apex in male with apical spines or plates (Figs 52-58)......7 Lateral pronotal margins smooth without tubercle; mesotibia at apex in male without apical spines or plates

Elytra without white or brown setae; body almost black, only 4 basal antennal segments and fore legs reddish orange..... Bruchus loti Elytra with white or brown setae (Figs 15-28); body not almost black .......8 Metatibia with mucro longer than lateral denticle (Figs 60, 61).................9 Metatibia with mucro shorter than lateral denticle (Fig. 59)

Four basal antennomeres, protibia and tarsi, part or all of mesotibia, and tarsi reddish orange; hind femur with long external tooth near apex; mesotibia at apex in male as Fig. 56.

Bruchus pisorum Five basal antennomeres reddish orange and rest black in female, antenna all reddish orange in male; hind femur with blunt external tooth near apex; mesotibia at apex in male as Fig. 58 Bruchus sibiricus Pygidium with white or brown, dense and long setae, with 2 subapical black spots (sometimes subapical spots indistinct as in Bruchus rufimanus) (Fig. 62); mesotibia at apex in male as Fig. 52, 54 or 57

Pygidium with gray, sparse and short setae, without subapical spot (Fig. 63); mesotibia at apex in male as Fig. 53 Bruchus atomarius Lateral pronotal margin with denticle at midpoint (Fig. 46); mesotibia at apex in male as Fig. 54 or 57

Lateral pronotal margin with denticle at $1 / 3$ near apex (Fig. 45); mesotibia at apex in male as Fig. 52

Bruchus affinis Lateral pronotal margin with prominent and sharp denticle; elytra with 3 rows of white stripes; pygidium with 2 distinct black subapical spots; mesotibia at apex in male as Fig. 54

Bruchus dentipes Lateral pronotal margin with blunt denticle; elytra varying from pattern of white spots on black background with short, yellowish brown stripes to variably distributed white spots; pygidium with 2 indistinct black subapical spots (Fig. 62); mesotibia at apex in male as Fig. 57

Bruchus rufimanus Body ovate; metatibia with 2 conspicuous apical spurs (Fig. 2); pronotum lateral margins complete Spermophagus sericeus Body suboval; metatibia without apical spur; pronotum lateral margins absent in apical half at least 
14 Hind femur with teeth both on inner and outer margins of ventral sulci (Figs 50, 51), sometimes denticle on distal margin blunt; posterior margin of pronotum bilobed at junction with scutellum and feebly gibbose there (Figs 9, 11) ...... 15

- $\quad$ Hind femur with outer margin of ventral sulci edentate; posterior margin of pronotum without feeble gibbose there............................................... 16

15 Elytral striae 3 and 4 each with prominent subbasal denticles on slight gibbose, antennae serrate in male.... Callosobruchus chinensis

- $\quad$ Elytral striae extending to basal margin without prominent denticles or gibbose, antennae smooth linear in male Callosobruchus maculatus

16 Hind femur ventrally with 3 distinct preapical teeth on inner margins of ventral sulci, proximal tooth much larger than others (Fig. 49)

\section{Acanthoscelides pallidipennis}

- $\quad$ Hind femur ventrally edentate or with 1 preapical tooth in internal margins of ventral sulci

17 Pygidium immaculate in male (Fig. 66) and with a pair of dark patches in female (Fig. 67) Megabruchidius dorsalis

- Pygidium without patches ...................................................... 18

18 Pronotum covered with orange pubescence (Fig. 7); pygidium tuberculate in female and smooth in male (Figs 64, 65) ...........Bruchidius tuberculicauda

- $\quad$ Pronotum covered with white pubescence (Fig. 5); pygidium not tuberculate

Bruchidius apicipennis

\section{Discussion}

Chinese literature on Bruchinae is out-of-date because of the recent changes in generic and tribal classification and description of new species (Borowiec 1987, Bouchard et al. 2011, Delobel and Legalov 2009). The majority of seed-beetle species in Xinjiang belong to the genera Bruchus and Kytorhinus. Of these, four species in this study are new records for China. These are Bruchus affinis, B. atomarius, B. loti and Kytorhinus kergoati. The first three have a wide distribution in the Palearctic Region. Only K. kergoati has been recorded in Tajikistan. Most of the new Bruchinae distribution records are found near the border. Acanthoscelides pallidipennis, Callosobruchus chinensis and $C$. maculatus are adventive species, so the extended human activity in Xinjiang is probably responsible for the beetle's extended distribution in this area too. Bruchidius apicipennis, B. tuberculicauda, Megabruchidius dorsalis, Rhaebus solskyi and Spermophagus sericeus appear to be eurytopic species found in a wide variety of habitats of the Palearctic Region.

The Bruchinae of Xinjiang remain relatively poorly investigated and it is likely that many additional species can still be found in the region. Further fieldwork is required to ascertain if the paucity of data is due to a genuinely small number of species, or the result of insufficient collection efforts. 


\section{Acknowledgement}

We are grateful to Dr. Liu Ning, Dr. Ren Li, Dr. Jens Prena and Mr. Yao Jian (Institute of Zoology, Chinese Academy of Science) for their help in many ways. We also thank Mr. Wang Jian, the director of Altay Forestry Science Research Institute, for providing host plant data in this study. Thanks to Prof. Jin Daochao, Prof. Li Zizhong and Dr. Yi Tianci (Guizhou University) for their guidance on taxonomy. This research was supported by the National Natural Sciences Foundation of China (31210103909/31172130//J1210002) and Animal Fauna Exploration of Altay, Border of China, Kazakhstan, Mongolia and Russia (2013FY110300).

\section{References}

Anton KW (2010) Catalogue of Palaearctic Coleoptera. Volume 6: Chrysomeloidea. Series Editor: Löbl I and Smetana A. Apollo Books, Stenstrup, 924 pp.

Borowiec L (1987) The genera of seed beetles (Coleoptera, Bruchidae). Polskie Pismo Entomologiczne 57: 3-207.

Borowiec L (1991) Revision of the genus Spermophagus Schoenherr (Coleoptera, Bruchidae, Amblycerinae). Genus (supplement): 1-198.

Bouchard P, Bousquet Y, Davies AE, Alonso-Zarazaga MA, Lawrence JF, Lyal CHC, Newton AF, Reid CAM, Schmitt M, Ślipiński SA, Smith ABT (2011) Family-group names in Coleoptera (Insecta). ZooKeys 88: 1-972. doi: 10.3897/zookeys.88.807

Delobel A, Legalov A (2009) A new species of Kytorhinus Fisch.-Wald. from western Pamir (Coleoptera: Bruchinae). Genus 20(1): 71-76.

Hoffmann A (1965) Ergebnisse der zoologischen Forschungen von Dr. Z. Kaszab in der Mongolei. 45. Bruchidae (Coleoptera). Folia Entomologica Hungarica NS 18: 563-566.

Kingsolver JM (2004) Handbook of the Bruchidae of the United States and Canada (Insecta, Coleoptera). United States Department of Agriculture, Agricultural Research Service, Technical Bulletin 1912: 1-324.

Lukjanovitsch F, Ter-Minassian M (1957) Seed beetles (Bruchidae). Fauna USSR, Coleoptera. Zool. Inst. Akad. Nauk SSR, Moscow, n.s. No.67. Fauna SSR, t. XXIV, n.1, 208 pp.

Southgate BJ (1979) Biology of the Bruchidae. Annual Review of Entomology 24(1): 449-473. doi: 10.1146/annurev.en.24.010179.002313

Sui JZ, Reyihanguli Ma DC (2001) Strengthening the inspection and quarantine of Callosobruchus maculatusc Fab.. Xinjiang Agricultural Sciences 38(5): 272. [in Chinese]

Tan JJ, Yu PY (1980) Economic insect fauna of China. Coleoptera. Chrysomeloidea (I) Science Press, Beijing, China, 213 pp. [in Chinese]

Xu YX (1991) Occurrence and control measures of Bruchid. Xinjiang Farmland Science and Technology 4: 24-26. [in Chinese]

Zhang CH, Hou JQ, Zhang L (1987) Note on Callosobruchus chinensis L. in Kumul Xinjiang. Xinjiang Agricultural Sciences (4): 23-24. [in Chinese] 